\title{
Somatic synonymous mutations in regulatory elements contribute to the genetic aetiology of melanoma
}

\author{
Di Zhang ${ }^{1,2}$ and Junfeng $\mathrm{Xia}^{2^{*}}$ \\ From The International Conference on Intelligent Biology and Medicine (ICIBM) 2019 \\ Columbus, OH, USA. 9-11 June 2019
}

\begin{abstract}
Background: Non-synonymous mutations altering tumor suppressor genes and oncogenes are widely studied. However, synonymous mutations, which do not alter the protein sequence, are rarely investigated in melanoma genome studies.

Methods: We explored the role of somatic synonymous mutations in melanoma samples from TCGA (The Cancer Genome Atlas). The pathogenic synonymous mutation and neutral synonymous mutation data were used to assess the significance of pathogenic synonymous mutations in melanoma likely to affect genetic regulatory elements using Fisher's exact test. Poisson distribution probabilities of each gene were used to mine the genes with multiple potential functional synonymous mutations affecting regulatory elements.

Results: Concentrating on five types of genetic regulatory functions, we found that the mutational patterns of pathogenic synonymous mutations are mostly involved in exonic splicing regulators in near-splicing sites or inside DNase I hypersensitivity sites or non-optimal codon. Moreover, the sites of miRNA binding alteration exhibit a significantly lower rate of evolution than other sites. Finally, 12 genes were hit by recurrent potentially functional synonymous mutations, which showed statistical significance in the pathogenic mutations. Among them, nine genes (DNAH5, ADCY8, GRIN2A, KSR2, TECTA, RIMS2, XKR6, MYH1, SCN1OA) have been reported to be mutated in melanoma, and other three genes (SLC9A2, CASR, SLC8A3) have a great potential to impact melanoma.
\end{abstract}

Conclusion: These findings confirm the functional consequences of somatic synonymous mutations in melanoma, emphasizing the significance of research in future studies.

Keywords: Melanoma, Synonymous mutations, Regulatory elements

\section{Background}

In recent years, significant efforts have been made in cancer genomic sequencing by a large consortium. For instance, The Cancer Genome Atlas (TCGA) aims to complete cancer genome sequencing for $>50$ types of cancers [1]. The Catalogue Of Somatic Mutations In Cancer (COSMIC) is recognized as the most comprehensive resource for exploring the impact of somatic mutations in cancers, which integrates TCGA and other

\footnotetext{
*Correspondence: jfxia@ahu.edu.cn

${ }^{2}$ Institutes of Physical Science and Information Technology, Anhui University, Hefei, Anhui, China

Full list of author information is available at the end of the article
}

data source [2]. These data are massive and available but finding the optimal way to mine the underlying information is significant and urgent.

Previous studies have indicated that somatic synonymous mutations are associated with a risk of cancer. Increasing evidence suggests that these synonymous mutations can lead to major splicing defects at the mRNA level or codon usage or function regions annotation features and consequently cause disease [3-6]. Systematic melanoma sequencing studies have identified multiple recurrent mutations that impose functional consequences on the progression of melanoma $[7,8]$.

(C) The Author(s). 2020 Open Access This article is distributed under the terms of the Creative Commons Attribution 4.0 International License (http://creativecommons.org/licenses/by/4.0/), which permits unrestricted use, distribution, and 
These studies mainly focus on non-synonymous mutations, ignoring the functional contribution of synonymous mutations to cancer. Recently, synonymous somatic mutations in BCL2L12 have been reported to increase BCL2L12 mRNA and protein levels during the development of melanoma [9]. It is important to note that synonymous mutations frequently act as driver mutations by altering splicing and other mechanisms in human cancers [4]. Gotea et al. systematically reviewed the direct impact of synonymous mutations on melanoma and other cancers via splicing, folding, stability, translation and miRNA binding [10]. It is becoming clear that synonymous mutations are not completely neutral, and therefore, their function needs to be further investigated.

To date, synonymous mutations in melanoma have rarely been investigated in published works. To test whether somatic synonymous mutations can induce the genetic aetiology of melanoma, we mainly focused on five mechanisms: splicing regulation, transcription factor binding, miRNA binding, codon optimality, and the RNA second structure to analyse their impact on melanoma (Fig. 1) [11]. We used the TCGA dataset in COSMIC database [2]. A functional impact score predicted by the FATHMM [12] algorithm was provided in COSMIC. Two groups of somatic synonymous mutations were compared: pathogenic synonymous mutations and the neutral mutations. Our findings present statistical evidence that pathogenic synonymous mutations in melanoma may act through changing exonic splicing regulators in near-splicing sites, altering DNase I hypersensitivity sites, and likely losing optimal codons. We then analysed the recurrent hit genes whose synonymous mutations are likely to contribute to the disease aetiology. We hope that our study will provide a new perspective in understanding somatic synonymous mutations in melanoma from the vast amount of cancer genome sequencing data.

\section{Results}

\section{Analysis of somatic synonymous mutational patterns in} melanoma

Somatic synonymous mutations in melanoma were obtained from COSMIC, and only the mutations occur in TCGA samples were obtained for further study. We analysed the distribution of the base changes in the 79,326 somatic synonymous mutations in melanoma. Figure 2 shows that the most frequently occurring base changes were $\mathrm{C} \rightarrow \mathrm{T}$ and $\mathrm{G} \rightarrow \mathrm{A}$. Though we have not performed on non-synonymous mutations, but from previous study, we known that they are also the two most frequently observed mutation types in non-synonymous mutations [13]. Two reasons may explain this result. First, the higher frequency of $\mathrm{C} \rightarrow \mathrm{T}$ and $\mathrm{G} \rightarrow \mathrm{A}$ base changes may be due to the epigenetic modification of DNA that commonly occurs at $\mathrm{CpG}$ dinucleotides. Second, the UV radiation (UVR) signature is related to $\mathrm{C} \rightarrow \mathrm{T}$ and $\mathrm{G} \rightarrow$ A transitions [14], which is consistent with previously documented findings that UVR exposure can increase melanoma risk [15].

\section{Pathogenic synonymous mutations in melanoma occur frequently via splicing regulation}

As described in the COSMIC, FATHMM scores greater than 0.7 are classified as pathogenic and scores less than

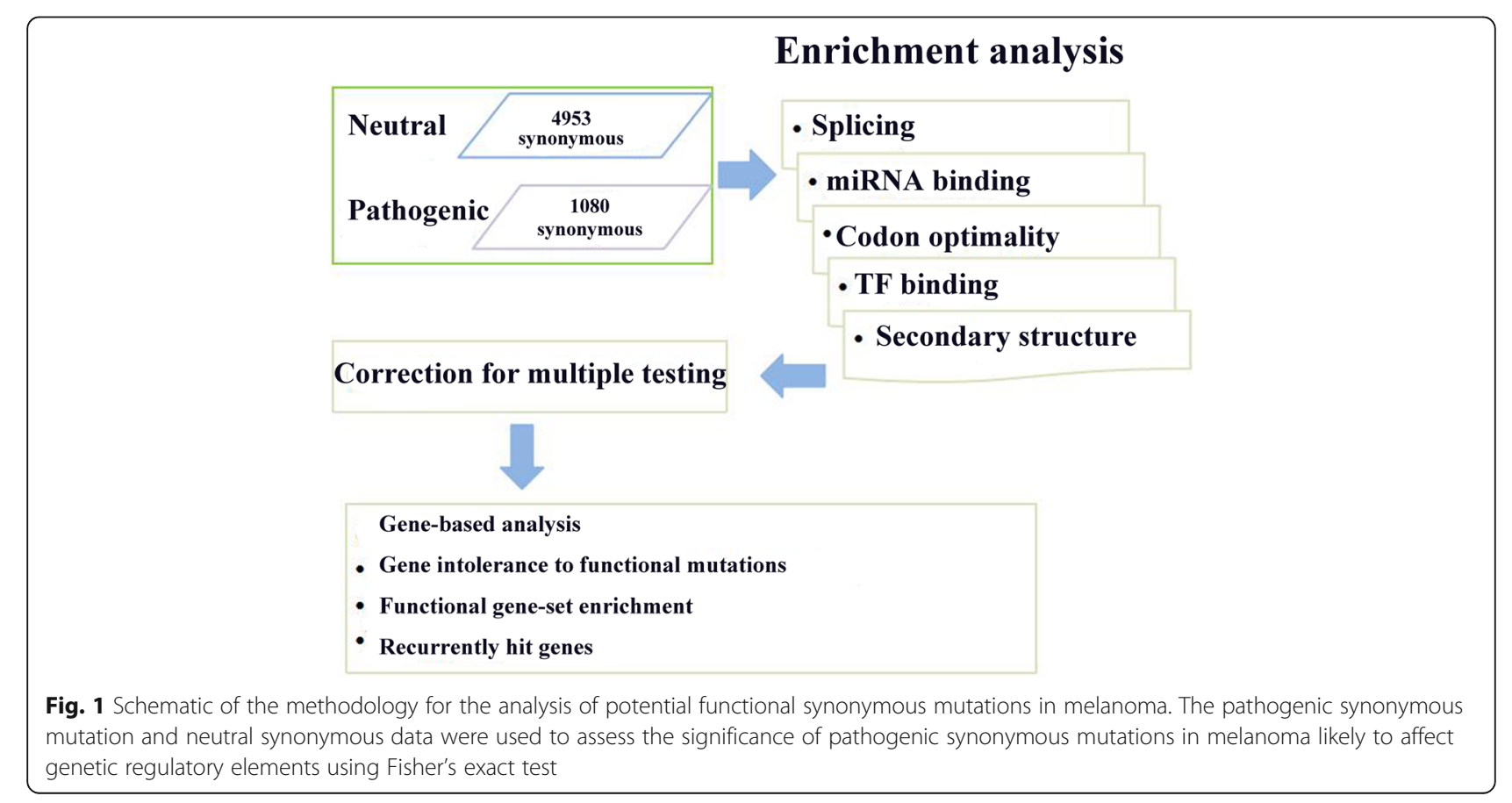




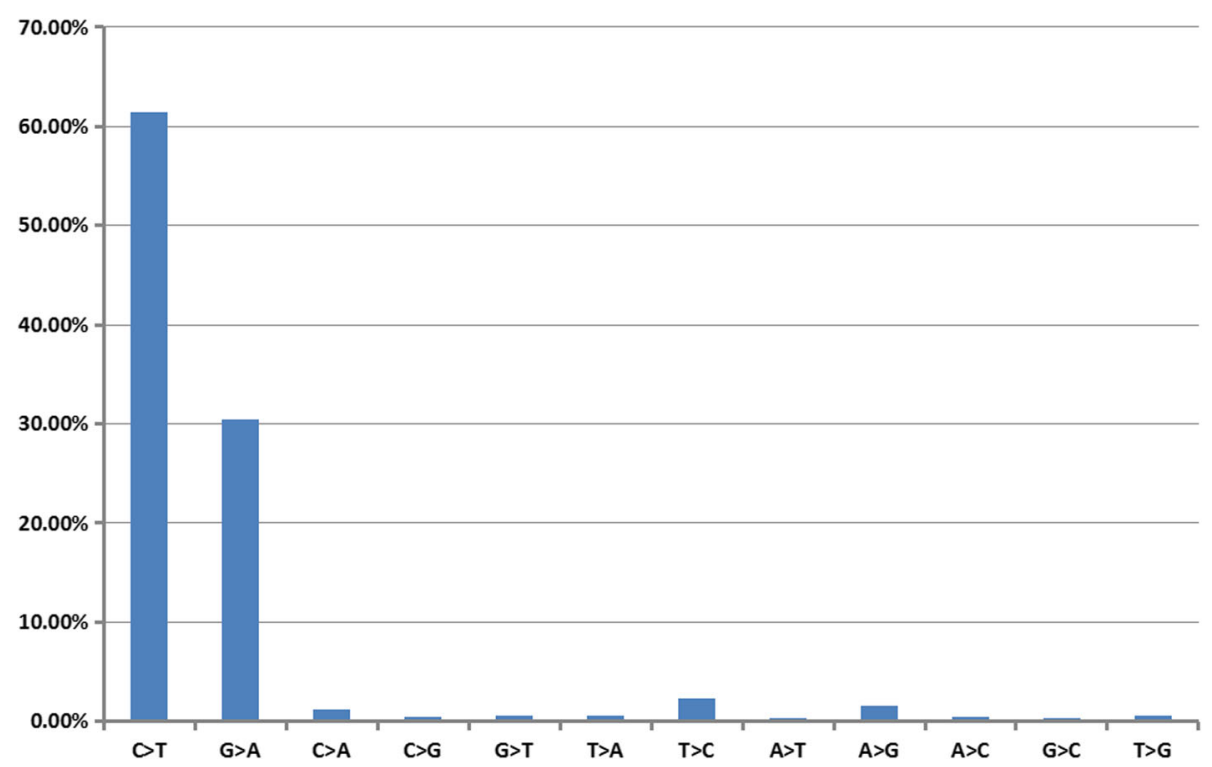

Fig. 2 Histogram showing the frequency of occurrence of each of the 12 possible base changes for all synonymous mutations

0.5 are labelled as neutral. To increase reliability, we kept only these mutations occurred in at least two samples for pathogenic synonymous mutations. To minimize false positives, we selected only those mutations occurred in one sample for neutral synonymous mutations. As a result of the described procedure, we obtained 1080 pathogenic synonymous mutations and 4953 neutral synonymous mutations in this study.

There has been a long-standing interest in identifying genes involved in the splice site. Recent work specifically described that synonymous mutations within $30 \mathrm{bp}$ of the nearest splice site tended to effect splicing regulation, and this area was defined as the near-splice site [4]. Therefore, in our data, we analysed synonymous mutations within the exonic near-splice site. Our study showed that near-splice sites of synonymous mutations occur nearly twice as often in pathogenic mutations than in neutral mutations $\left(p=5.89 \times 10^{-13}\right.$, odds ratio [OR] $=$ 1.76, 310 pathogenic mutations, 921 neutral mutations, Fisher's exact test). This result is inconsistent with Supek's study [4]. From the cumulative distributions of pathogenic synonymous mutations and neutral synonymous mutations (Fig. 3a), we can see that the distance to the nearest splicing site is closer in the pathogenic mutations than in the neutral mutations. Although Supek's study reported that the difference in cumulative distributions is near $30 \mathrm{bp}$ [4], in our study, the difference in cumulative distributions between these two

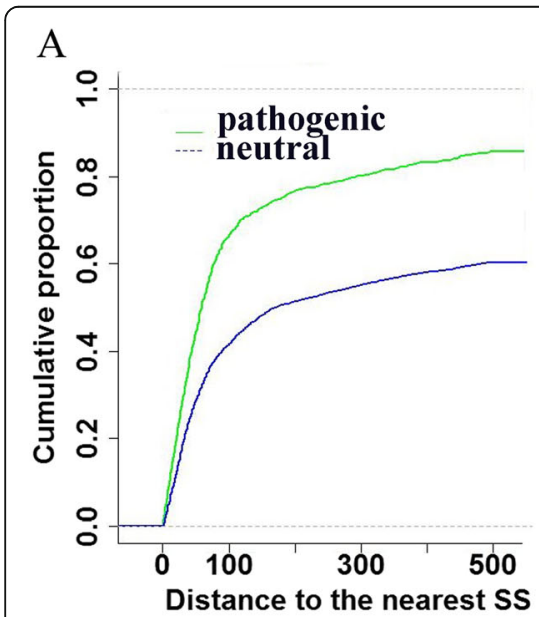

B

Fig. 3 a Cumulative proportion curves for the distance to the nearest splice site. The proportion of the synonymous mutations with a distance to the nearest splice sites less than the number indicted in the $X$ axis was plotted on the $Y$ axis. The green line represents the pathogenic synonymous mutations, while the blue line represents the neutral synonymous mutations. $\mathbf{b}$ Enrichment of synonymous mutations near splice sites (odds ratios shown above) 
classes of synonymous mutations is $31-70 \mathrm{bp}$ (Fig. 3b). This may be because our definition of pathogenic mutations was stricter.

Synonymous mutations can influence pre-mRNA splicing by modulating ESS (Exonic Splicing Silencer) and ESE (Exonic Splicing Enhancer) motifs [16]. We referenced the ESE from the RESCUE-ESE website and ESS from the FAS-ESS website (see Methods section). Then, we mapped the synonymous mutations to the list of ESEs and ESSs (402 ESEs and 316 ESSs were collected in our study). From Fig. 4a, we can see that pathogenic synonymous mutations with ESR (exonic splicing regulator) change (i.e., ESE gain, ESS gain, ESE loss, ESS loss) occur in the near-splicing site more often than neutral synonymous mutations $\left(p=1.28 \times 10^{-7}, \mathrm{OR}=1.72\right.$, Fisher's exact test). This in support of the research that pathogenic synonymous mutations are deleterious owing to disrupting ESR sequences, with a direct result in aberrant gene splicing [17].

\section{Pathogenic synonymous mutations in melanoma do not frequently alter mRNA secondary structure or miRNA target sites}

Next, we consider whether pathogenic synonymous mutations contribute to melanoma by influencing the mRNA secondary structure, which also changes translation efficiency [18]. We used RNAsnp software, which uses the local regions of maximal structural change between the mutant and wild-type to predict the synonymous mutation effects on mRNA secondary structure [19]. However, we found that pathogenic synonymous mutation effects on mRNA folding do not seem to be common mechanisms in melanoma (see Fig. 4b). While most common mutation associated with cystic fibrosis affecting the mRNA secondary structure [10]. It remains to be determined whether pathogenic synonymous mutations affect mRNA secondary structures depending on disease category.

Synonymous mutations interfere with miRNA binding, which can alter the nucleotide sequences targeted by miRNAs [10]. However, we found that the pathogenic synonymous mutations effects on miRNA binding do not seem to be common mechanisms in melanoma (see Fig. 4c). The nucleotide sequences of the putative miRNA binding site exhibit a significantly lower rate of evolution at the synonymous mutation sites than other gene sequences [20]. In our study, we considered whether the site of miRNA binding alteration presents high conservation. As expected, the conservation of the site with miRNA binding alteration is higher than the site with no miRNA binding alteration $(p=0.028)$ in pathogenic synonymous mutations. Therefore, pathogenic synonymous mutations do not seem to affect RNA secondary structure or miRNA binding sites, although they may still be vital in individual cases [9].

\section{Pathogenic synonymous mutations in melanoma decrease codon optimality}

Pathogenic synonymous mutations affect gene activity through diverse molecular mechanisms [21]. We evaluated whether pathogenic synonymous mutations in melanoma tend to increase or decrease the use of optimal codons, thereby affecting translation efficiency [22]. When a synonymous mutation changes an optimal codon to a non-optimal codon, it may introduce a pause in the translation machinery. Then, a pathological condition will be decided by the degree of the affected functional protein. As expected, pathogenic synonymous mutations show a general tendency towards less optimal codons, while gain of the optimal codons is seen in neutral synonymous mutations (Fig. 4d).

\section{Pathogenic synonymous mutations in melanoma are likely located in DNase I hypersensitive sites (DHSs)}

Functional mutations are more likely to target transcription factor (TF) binding sites and are especially located in DNase I hypersensitive sites (DHSs), which are open chromatin regions for proteins to approach [11]. Chromatin regions of DHSs and TF are functionally related to transcriptional activity and necessary for the binding of proteins such as transcription factors. According to genome-wide association studies, DHSs are significantly enriched for transcriptional signals [23]. We downloaded the TF binding site and DHS information from the ENCODE (Encyclopedia of DNA Elements) experiment matrix. We analysed whether pathogenic synonymous mutations in melanoma was preferentially found in TF and DHS and found a significant target in DHS but no significant target in TF compared to the neutral synonymous mutations.

\section{Potentially functional synonymous mutations in melanoma are involved in transport pathways}

Through the above analyses, we found that the pathogenic synonymous mutations are enriched in three aspects, including exonic splicing regulators within the near-splicing site, DNase I hypersensitivity sites, and loss of optimal codons. If one of the pathogenic synonymous mutations is in accord with one of the three aspects, we defined it as a potentially functional synonymous mutation. Among the 1080 pathogenic synonymous mutations, 713 mutations were functional synonymous mutations, which presents $11.8 \%$ (713/6033) of total synonymous mutations (1080 pathogenic and 4953 neutral synonymous mutations) used in this study. Functional gene enrichment analysis was performed using the online service website DAVID [24]. As shown in Fig. 5, 


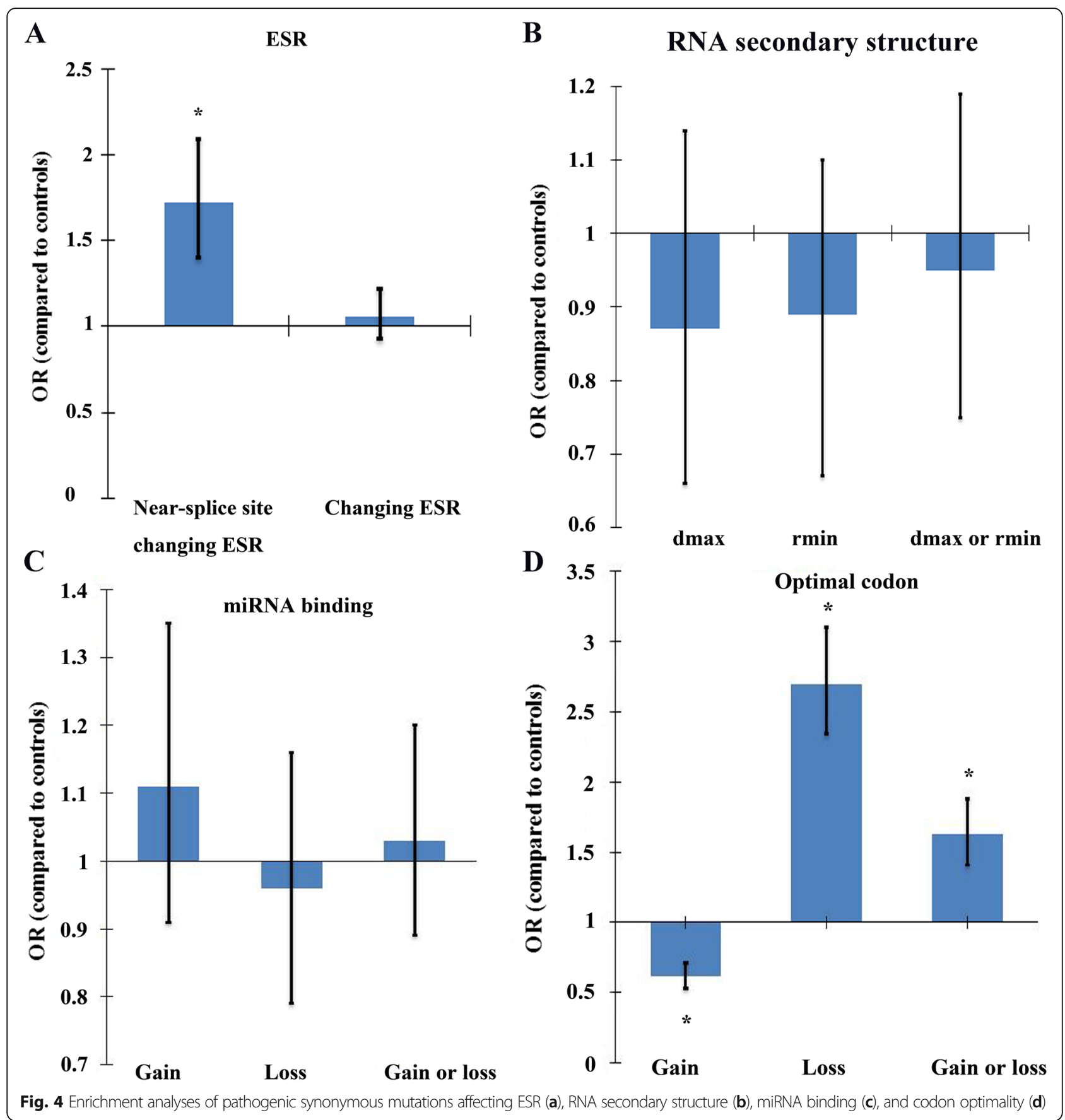

these genes were significantly enriched in genes associated with transport pathways (e.g., "metal ion transport", Benjamini-Hochberg corrected $p$ value $\left[\mathrm{p}_{\mathrm{BH}}\right]=2.48 \times$ $10^{-8}$; "cation transport"), neuronal function (e.g., "neurological system process", $\mathrm{p}_{\mathrm{BH}}=6.45 \times 10^{-8}$ ), and signalling pathways (e.g., "cell-cell signalling", $\mathrm{p}_{\mathrm{BH}}=$ $\left.6.25 \times 10^{-4}\right)$. In recent years, it has become increasingly clear that ion transporters (such as GO:0006811 ion transport, seen in Fig. 5) is an important mechanism in the development of drug resistance [25]. We infer that the transport pathway may be related to drug resistance, and suggest that they may have strong potential as diagnostic and therapeutic targets in melanoma treatment.

Candidate genes that are recurrently hit by potentially functional synonymous mutations in melanoma

The identification of these functional synonymous mutations based on high recurrence can assist in detecting new genes that participate in melanoma. In our study, we assessed the statistical significance of observing potential 


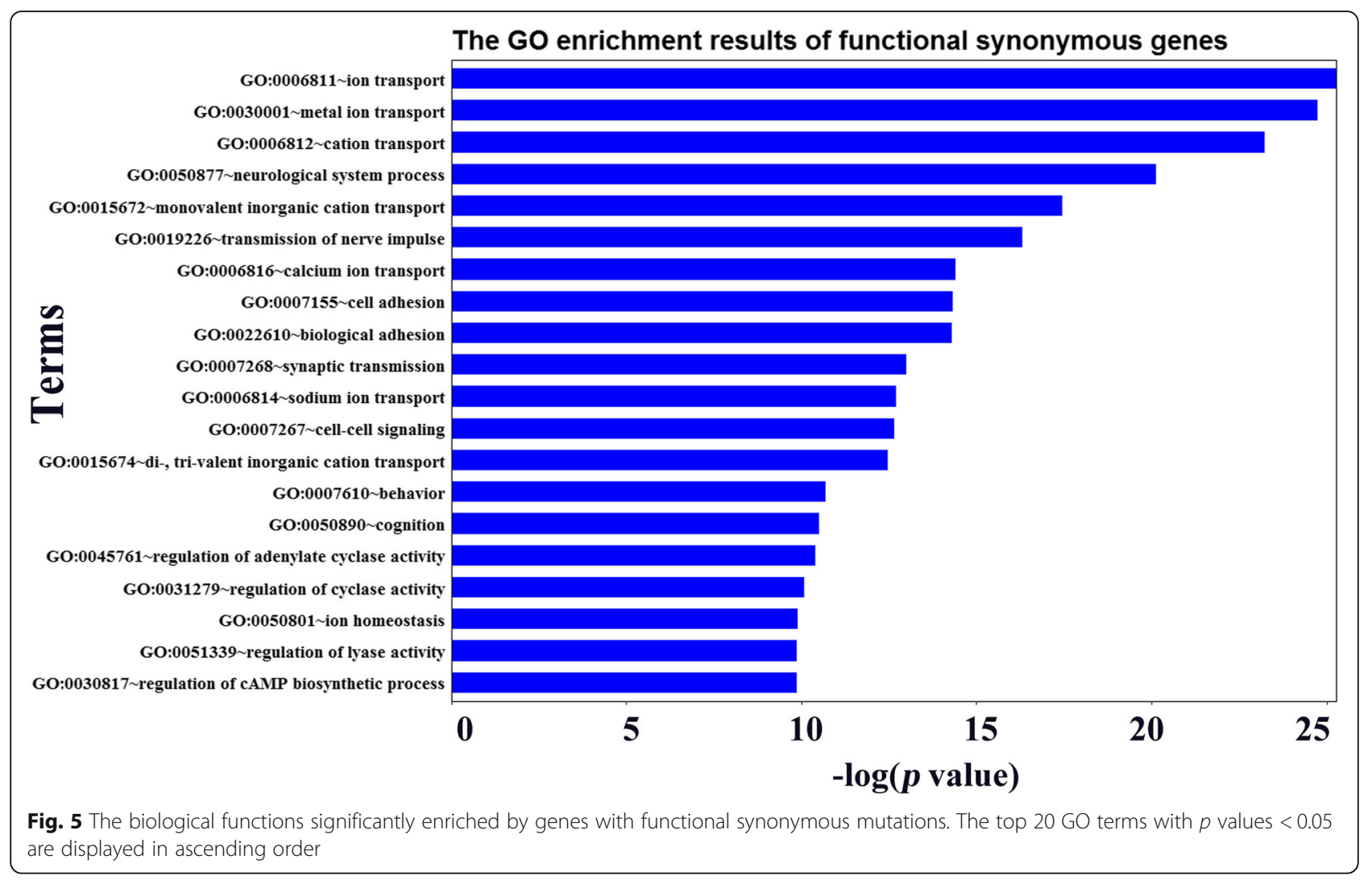

functional synonymous mutations for each gene by analysing high-quality variant calls across 60,706 human exomes [26]. Previous research indicates that the genome-wide significance threshold is $2.74 \times 10^{-6}$ [27]. In our study, the probability of observing potential functional synonymous mutations was calculated by Poisson distribution. Table 1 shows significant genes with multiple synonymous mutations across 341 melanoma patients. Notably, not all genes with many synonymous mutations have significant $p$ values. For example, TTN has 5 potential functional synonymous mutations in the case group but does not have a nominally significant $p$ value $\left(p\right.$ value $\left.=7.11 \times 10^{-6}\right)$, owing to the long length of the gene. Among these genes identified in this study, 12 genes are hit by potential functional synonymous mutations in melanoma (Table 1). Of these, DNAH5 mutations in melanoma samples correlate with a negative outcome [28]. ADCY8 mutations were previously identified in melanoma patients [29]. KSR2 have been shown to have pro-tumorigenic roles [30].GRIN2A harbors 5 potential functional synonymous mutations and encodes the NMDA (N-methyl-(D)-aspartic acid) receptor subunit, which bears the agonist binding site for glutamate [31]. It has been reported that 11 somatic mutations in GRIN2A were involved in melanoma [32]. The $p$ value $\left(9.99 \times 10^{-12}\right)$ for this gene is less than the genome-wide significance threshold. TECTA has significantly more potential functional synonymous mutations than expected $\left(p=1.78 \times 10^{-8}\right)$, in which mutations occurred in melanoma [33]. In our study, for the TECTA gene, 2 mutations were found in the nearsplice site and accompanied by ESR changes, and 2 mutations were found in DHSs. In addition, both GRIN2A and TECTA were among the most intolerant genes according to the RVIS (Residual Variation Intolerance Score), indicating that the variants within these genes are under natural selection and may be more likely to influence melanoma. RIMS2 significantly upregulated in monosomy 3 melanomas [34]. XKR6 mutations already reported in cutaneous malignant melanoma cell lines [35]. SCN10A commonly mutated among TCGA melanoma samples [28]. MYH1 has recently been shown to be upregulated in melanoma [36]. We also identified mutations in three genes (SLC9A2, CASR, SLC8A3) never reported in melanoma, which might deserve further investigations.

\section{Discussion}

The first theme of this study is to investigate somatic synonymous mutations in regulatory elements contribute to the genetic aetiology of melanoma. From our analyses, we found that mutations with near-splicing site mutations changing ESR, targeting DHI sites and preferentially using non-optimal codons are significantly enriched in pathogenic synonymous mutations. After performing gene functional enrichment, we obtained several potential functional synonymous mutations 
Table 1 Genes with recurrent synonymous mutations potentially affecting regulatory elements

\begin{tabular}{|c|c|c|c|c|c|}
\hline Gene & Gene length & Putative mechanisms $^{a}$ & $\begin{array}{l}\text { Number of functional } \\
\text { synonymous mutations }\end{array}$ & $\begin{array}{l}\text { Number of expected } \\
\text { synonymous mutations }\end{array}$ & $p$ values \\
\hline DNAH5 & 13,875 & $\begin{array}{l}\text { disrupt ESR sequence in near SS, alter DHS, and } \\
\text { decrease the use of optimal codons }\end{array}$ & 13 & 0.037106647 & $3.92 \times 10^{-29}$ \\
\hline ADCY8 & 3756 & $\begin{array}{l}\text { disrupt ESR sequence in near SS, alter DHS, and } \\
\text { decrease the use of optimal codons }\end{array}$ & 5 & 0.014354406 & $5.02 \times 10^{-12}$ \\
\hline GRIN2A & 4395 & $\begin{array}{l}\text { disrupt ESR sequence in near SS, alter DHS, and } \\
\text { decrease the use of optimal codons }\end{array}$ & 5 & 0.01648022 & $9.99 \times 10^{-12}$ \\
\hline SLC9A2 & 2439 & $\begin{array}{l}\text { disrupt ESR sequence in near SS, alter DHS, and } \\
\text { decrease the use of optimal codons }\end{array}$ & 4 & 0.008004311 & $1.7 \times 10^{-10}$ \\
\hline CASR & 3267 & alter DHS, decrease the use of optimal codons & 4 & 0.011093165 & $6.25 \times 10^{-10}$ \\
\hline KSR2 & 2853 & $\begin{array}{l}\text { disrupt ESR sequence in near SS, alter DHS, and } \\
\text { decrease the use of optimal codons }\end{array}$ & 4 & 0.012140187 & $8.96 \times 10^{-10}$ \\
\hline MYH1 & 5820 & $\begin{array}{l}\text { disrupt ESR sequence in near SS, alter DHS, and } \\
\text { decrease the use of optimal codons }\end{array}$ & 4 & 0.014354315 & $1.75 \times 10^{-9}$ \\
\hline TECTA & 6468 & $\begin{array}{l}\text { disrupt ESR sequence in near SS, alter DHS, and } \\
\text { decrease the use of optimal codons }\end{array}$ & 4 & 0.02571247 & $1.78 \times 10^{-8}$ \\
\hline SLCBA3 & 2784 & $\begin{array}{l}\text { disrupt ESR sequence in near SS, decrease the use of } \\
\text { optimal codons }\end{array}$ & 3 & 0.00836151 & $9.68 \times 10^{-8}$ \\
\hline RIMS2 & 4050 & alter DHS, decrease the use of optimal codons & 3 & 0.009850803 & $1.58 \times 10^{-7}$ \\
\hline XKR6 & 1926 & alter DHS & 3 & 0.010850219 & $2.11 \times 10^{-7}$ \\
\hline SCN1OA & 5871 & alter DHS, decrease the use of optimal codons & 3 & 0.016168958 & $6.96 \times 10^{-7}$ \\
\hline
\end{tabular}

${ }^{a} E S R$ : exonic splicing regulators, SS: splicing sites, DHS: DNase I hypersensitivity sites

related to biological processes such as transporter and signalling pathway that have previously been implicated in melanoma. Pathogenic synonymous mutations may participate in transport pathway and might be related to drug resistance.

Another theme of this study is the estimation of the number of genes in which functional synonymous mutations affecting regulatory elements in melanoma. Biases in functional mutation could make some tissues more prone to accumulating functional mutations in certain genes (e.g., exposure to mutational processes). By comparing the number of expected synonymous mutations, we inferred 12 candidate genes across melanoma. Most of these genes generally agree with previously reported genes in melanoma. Remarkably, there is a connection between candidate genes and intolerant gene under positive selection. Recent analysis of synonymous and non-synonymous mutations in tumor genomes has revealed that positive selection accelerates the fixation of somatic mutations in a gene-specific manner $[37,38]$. Our results imply that selection pressures during melanoma progress is a vital event.

The excesses of potential functional synonymous mutations across gene sets were evaluated by calculating the significance for individual genes. Though the potential functional synonymous mutations take a small part of synonymous mutations, a few mutations tend to drive melanoma. It is likely that pathogenic synonymous mutations affect regulatory elements. These mutations can explain a substantial part of melanoma liability.

\section{Conclusion}

In summary, we used existing published TCGA data to identify pathogenic synonymous mutations in melanomas. Future functional and preclinical studies are needed to be conducted to determine whether these synonymous mutations are pathogenic or neutral mutations. Going forward, the study presented here may pave the way for analysing synonymous mutation data in other types of cancer, like lung cancer.

\section{Methods \\ Data collection}

Published somatic synonymous mutations in melanoma were collected from the TCGA data in COSMIC (v80 release). For gene with multiple transcripts, only the longest transcript was remained. The FATHMM (Functional Analysis Through Hidden Markov Models) scores predicting functional consequences of somatic synonymous mutations were also obtained from the COSMIC.

\section{Analysis of synonymous mutations changing splicing regulation}

The annotation for the distance to the nearest splice site for each synonymous mutation was obtained by SeattleSeq annotation 138 online website (http://snp.gs.washington.edu/SeattleSeqAnnotation138/). 
To investigate whether the near-splice site synonymous mutations tend to affect exonic splicing regulators (exonic splicing enhancer (ESE) and silencer (ESS)), we used the following lists of predicted ESEs and ESSs: 1) top 200 ESEs with "ESEseq" descending order and top 200 ESSs with "ESSseq" ascending order in the attachment of literature, in which ESE and ESS were experimentally tested [39]. 2) A total of 238 ESEs were curated from RESCUE-ESE Web Server (http://hollywood.mit. edu/burgelab/rescue-ese), which provides a hybrid computational/experimental method to identify candidate ESEs in vertebrate exons. 3) A total of 176 ESSs were curated from FAS-ESS Web Server (http://hollywood.mit. edu/fas-ess), which uses a splicing reporter system to identify ESSs. The list of ESEs was the union set of the above two resources, and similarly, the list of ESSs was the union set of the above two resources (in total, 402 ESEs and 316 ESSs). We compared the set of sequences consisting of the mutated site with $6 \mathrm{bp}$ windows $(-5 \sim 0$, $-4 \sim+1,3 \sim+2,2 \sim+3,1 \sim+4$, and $0 \sim+5$ when the mutation site is at position 0 ) to the reference lists of ESE and ESS sequences, and checked whether each synonymous mutation caused ESE or ESS events. The reference site was calculated similarly. Four types (ESE gain, ESE loss, ESE gain or ESS loss) of ESR change were considered for all synonymous mutations.

SPANR (Splicing-based Analysis of Variants) was adopted to measure the impact of synonymous mutations on splicing regulation [40] and evaluations were performed with all the synonymous mutations.

\section{DNase I hypersensitive sites (DHS) analysis}

The mapped read BAM files in Encyclopedia of DNA Elements (ENCODE) were downloaded from http://genome.ucsc.edu/ENCODE/downloads.html, which were used for DHS annotations. The raw downloaded DHS data contains the defining DHS peaks and footprints. Synonymous mutations that lied near DHS sites were not considered as overlapping with DHS.

\section{miRNA binding site analysis}

The FASTA file of high confidence miRNAs was downloaded from miRBase (http://www.mirbase.org/blog/2 014/07/high-confidence-mirna-set-available-for-mir-

base-21/). Then, we extracted the miRNA seed sequences. When the first base was adenine, position 2-7 sequences were selected. Base A appears to anchor the very $3^{\prime}$ terminus of the miRNA complementary site, which suggests that requiring a 6-nt seed match followed by this "A anchor" would increase the specificity of target prediction [41]. Otherwise, position 2-8 sequences were selected. We compared the corresponding mutation sequences (7-bp windows $(-6 \sim 0,-5 \sim+1,4 \sim+2$, $3 \sim+3,2 \sim+4,-1 \sim+5$, and $0 \sim+6$ when the mutation site is at position 0) and the miRNA seed sequences, and evaluated whether a synonymous mutation can cause at least on loss or gain in the miRNA binding site.

\section{Optimal codon analysis}

To study whether synonymous mutations lead to a gain or loss of the optimal codon events, we downloaded the previously defined optimal codons from Table S6 of literature [4]. For each synonymous mutation, the corresponding codons for reference and variant alleles were obtained using the variant effect predictor tool [42].

\section{RNA secondary structure analysis}

To evaluate the impact of synonymous mutations on RNA secondary structures, we input sequence files and lists of mutations to be tested into the RNAsnp software [19] with default parameters. An option to run RNAsnp on the genome sequence is to split the input sequence chromosome-wise, which can be done similarly for the synonymous mutations (without chromosome number as prefix). The maximum Euclidean distance and minimum Pearson correlation coefficient were the two methods used to calculate the $p$ value.

\section{Mutations in genes intolerant to functional variation analysis}

The genes intolerant to functional variation were downloaded from the literature [43]. We considered the top $25 \%$ percentage of the genes as "intolerant" genes. Thus, mutations corresponding to these intolerant genes have RVIS (Residual Variation Intolerance Score) information. Mutations without RVIS information were excluded from the analysis.

\section{Evolutionary conservation analysis}

Phylop.mammalian conservation scores for the variant were obtained from MyVariant.info [44]. This score is positively correlated with evolutionary conservation.

\section{Gene functional enrichment analysis for the genes with potential functional synonymous mutations}

Genes with potential functional synonymous mutations were input into the DAVID online web server (V6.7) [24]. The biological process was the category of functional annotations. $\mathrm{B}-\mathrm{H}$ was used to adjust the $p$ value. Terms with $\leq 5$ hit counts in the query list were filtered.

\section{Recurrent gene analysis}

To mine the genes with multiple potential functional synonymous mutations affecting regulatory elements, we downloaded the gene's corresponding probability of synonymous mutations according to a recently developed pipeline for the interpretation of synonymous mutations 
[27]. The number of expected synonymous mutations $\lambda$ in each gene is calculated as follows:

$$
\lambda=2 * R * S,
$$

where $R$ represents the mutation rate for each synonymous mutation [26], $S$ represent the number of samples (341 samples in our study).

Poisson distribution probabilities of each gene $P$ was calculated as follows [27]:

$$
\mathrm{P}(\mathrm{X}=\kappa)=\frac{\lambda^{\kappa}}{\kappa !} e^{-\lambda}
$$

where $k$ represents the number of functional synonymous mutations in corresponding gene, which was decided by the number of functional synonymous mutations in the gene. Only the Poisson distribution probabilities $p$ of each gene less than $1 \times 10^{-6}$ was defined as recurrent genes. The $p$-values were not adjusted for multiple comparisons.

\section{Abbreviations}

COSMIC: The Catalogue Of Somatic Mutations In Cancer; DHSs: DNase I hypersensitive sites; ENCODE: Encyclopedia of DNA Elements; ESE: Exonic Splicing Enhancer; ESR: Exonic Splicing Regulator; ESS: Exonic Splicing Silencer; FATHMM: Functional Analysis through Hidden Markov Models; RVIS: Residual Variation Intolerance Score; TCGA: The Cancer Genome Atlas; TF: Transcription Factor

\section{Acknowledgements}

The authors thank all members of our laboratory for their valuable discussions.

\section{About this supplement}

This article has been published as part of BMC Medical Genomics Volume 13 Supplement 5, 2020: The International Conference on Intelligent Biology and Medicine (ICIBM) 2019: Computational methods and application in medical genomics (part 1). The full contents of the supplement are available online at https://bmcmedgenomics.biomedcentral.com/articles/supplements/ volume-13-supplement-5.

\section{Authors' contributions}

DZ collected the datasets, performed the analysis, and drafted the manuscript. JX designed the study, performed the analysis, and drafted the manuscript. All authors read and approved the final manuscript.

\section{Funding}

Publication costs are funded by the National Natural Science Foundation of China (U19A2064, 61672037, and 11835014) and in part by the Young Wanjiang Scholar Program of Anhui Province, the Anhui Provincial Outstanding Young Talent Support Plan (gxyqZD2017005), and the Recruitment Program for Leading Talent Team of Anhui Province (2019-16). The funding bodies played no role in the design of the study and collection, analysis, and interpretation of data and in writing the manuscript.

\section{Availability of data and materials}

The datasets analysed during the current study are available in the COSMIC database (https://cancer.sanger.ac.uk/cosmic), ENCODE datasets (http:// genome.ucsc.edu/ENCODE), and miRBase database (http://www.mirbase.org). All the other datasets analysed during this study are openly available online and URLs are provided in this published article.

Ethics approval and consent to participate Not applicable.
Consent for publication

Not applicable.

\section{Competing interests}

The authors declare that they have no competing interests.

\section{Author details}

${ }^{1}$ College of information science and engineering, Shaoguan University,

Shaoguan, Guangdong, China. ${ }^{2}$ Institutes of Physical Science and Information Technology, Anhui University, Hefei, Anhui, China.

Published: 3 April 2020

\section{References}

1. Gonzalezperez A, Mustonen V, Reva B, Ritchie GRS, Creixell P, Karchin R, Vazquez M, Fink JL, Kassahn KS, Pearson JV. Computational approaches to identify functional genetic variants in cancer genomes. Nat Methods. 2013; 10(8):723-9.

2. Forbes SA, Beare D, Gunasekaran P, Leung K, Bindal N, Boutselakis $H$, Ding M, Bamford S, Cole C, Ward S. COSMIC: exploring the world's knowledge of somatic mutations in human cancer. Nucleic Acids Res. 2014;43(D1):D805-11.

3. Pecce $V$, Sponziello M, Damante G, Rosignolo F, Durante C, Lamartina L, Grani G, Russo D, di Gioia CR, Filetti S. A synonymous RET substitution enhances the oncogenic effect of an in-cis missense mutation by increasing constitutive splicing efficiency. PLoS Genet. 2018;14(10):e1007678.

4. Supek F, Miñana B, Valcárcel J, Gabaldón T, Lehner B. Synonymous mutations frequently act as driver mutations in human cancers. Cell. 2014; 156(6):1324-35.

5. Shi F, Yao Y, Bin Y, Zheng C-H, Xia J. Computational identification of deleterious synonymous variants in human genomes using a feature-based approach. BMC Med Genet. 2019;12(1):12.

6. Wang $C$, Zhou W, Huang $Y$, Yin $H$, Jin $Y$, Jia Z, Zhang A, Liu Z, Zheng B. Presumed missense and synonymous mutations in ATP 7B gene cause exon skipping in Wilson disease. Liver Int. 2018;38(8):1504-13.

7. Hodis E, Watson IR, Kryukov GV, Arold ST, Imielinski M, Theurillat J, Nickerson E, Auclair D, Li L, Place CS. A landscape of driver mutations in melanoma. Cell. 2012:150(2):251-63.

8. Krauthammer M, Kong YL, Ha BH, Evans P, Bacchiocchi A, Mccusker JP, Cheng E, Davis MJ, Goh G, Choi M. Exome sequencing identifies recurrent somatic RAC1 mutations in melanoma. Nat Genet. 2012:44(9):1006-14.

9. Gartner JJ, Parker SCJ, Prickett TD, Duttonregester K, Stitzel ML, Lin JC, Davis S, Simhadri $\mathrm{VL}$, Jha S, Katagiri NH. Whole-genome sequencing identifies a recurrent functional synonymous mutation in melanoma. Proc Natl Acad Sci U S A. 2013;110(33):13481-6.

10. Gotea V, Gartner JJ, Qutob N, Elnitski L, Samuels Y. The functional relevance of somatic synonymous mutations in melanoma and other cancers. Pigment Cell Melanoma Res. 2015;28(6):673-84.

11. Takata A, lonita-Laza I, Gogos JA, Xu B, Karayiorgou M. De novo synonymous mutations in regulatory elements contribute to the genetic etiology of autism and schizophrenia. Neuron. 2016;89(5):940-7.

12. Shihab HA, Gough J, Cooper DN, Day INM, Gaunt TR. Predicting the functional consequences of cancer-associated amino acid substitutions. Bioinformatics. 2013:29(12):1504-10.

13. Stiller M, Sucker A, Griewank KG, Aust D, Baretton GB, Schadendorf D, Horn $\mathrm{S}$. Single-strand DNA library preparation improves sequencing of formalinfixed and paraffin-embedded (FFPE) cancer DNA. Oncotarget. 2016;7(37): $59115-28$.

14. Greenman C, Stephens P, Smith R, Dalgliesh GL, Hunter C, Bignell G, Davies $\mathrm{H}$, Teague J, Butler A, Stevens C. Patterns of somatic mutation in human cancer genomes. Nature. 2007;446(7132):153.

15. Mukhopadhyay P, Ferguson B, Muller H, Handoko H, Walker G. Murine melanomas accelerated by a single UVR exposure carry photoproduct footprints but lack UV signature C> T mutations in critical genes. Oncogene. 2016;35(25):3342-50

16. Cartegni L, Chew SL, Krainer AR. Listening to silence and understanding nonsense: exonic mutations that affect splicing. Nat Rev Genet. 2002; 3(4):285.

17. Caceres EF, Hurst LD. The evolution, impact and properties of exonic splice enhancers. Genome Biol. 2013;14(12):1-18. 
18. Goodman DB, Church GM, Kosuri S. Causes and effects of N-terminal codon bias in bacterial genes. Science. 2013;342(6157):475-9.

19. Sabarinathan R, Tafer H, Seemann SE, Hofacker IL, Stadler PF, Gorodkin J. RNAsnp: efficient detection of local RNA secondary structure changes induced by SNPs. Hum Mutat. 2013;34(4):546-56.

20. Hurst LD. Preliminary assessment of the impact of MicroRNA-mediated regulation on coding sequence evolution in mammals. J Mol Evol. 2006; 63(2):174-82.

21. Sauna ZE, Kimchi-Sarfaty C. Understanding the contribution of synonymous mutations to human disease. Nat Rev Genet. 2011;12(10):683.

22. Drummond DA, Wilke CO. Mistranslation-induced protein Misfolding as a dominant constraint on coding-sequence evolution. Cell. 2008;134(2): 341-52.

23. Maurano MT, Humbert R, Rynes E, Thurman RE, Haugen E, Wang H, Reynolds AP, Sandstrom R, Qu H, Brody J. Systematic localization of common disease-associated variation in regulatory DNA. Science. 2012; 337(6099):1190-5.

24. Huang DW, Sherman BT, Lempicki RA. Systematic and integrative analysis of large gene lists using DAVID bioinformatics resources. Nat Protoc. 2009;4(1):44

25. Hoffmann EK, Lambert IH. Ion channels and transporters in the development of drug resistance in cancer cells. Philos Trans R S B Biol Sci. 2014:369(1638):20130109.

26. Lek M, Karczewski K, Minikel E, Samocha K, Banks E, Fennell T, O'DonnellLuria A, Ware J, Hill A, Cummings B. Analysis of protein-coding genetic variation in 60,706 humans. Nature. 2016;536(7616):285-91.

27. Samocha KE, Robinson EB, Sanders SJ, Stevens C, Sabo A, McGrath LM, Kosmicki JA, Rehnström K, Mallick S, Kirby A. A framework for the interpretation of de novo mutation in human disease. Nat Genet. 2014; 46(9):944.

28. Yavorski JM, Stoll RJ, Samy MD, Mauro JA, Blanck G. Identification of Sets of Cytoskeletal Related and Adhesion-related Coding Region Mutations in the TCGA Melanoma Dataset that Correlate with a Negative Outcome. Curr Genomics. 2017;18(3):287-97.

29. Kontogianni G, Piroti G, Maglogiannis I, Chatziioannou A, Papadodima O Dissecting the mutational landscape of cutaneous melanoma: an Omic analysis based on patients from Greece. Cancers. 2018;10(4):96.

30. Fernandez MR, Henry MD, Lewis RE. Kinase suppressor of Ras 2 (KSR2) regulates tumor cell transformation via AMPK. Mol Cell Biol. 2012;32(18): 3718-31.

31. Johnson JW, Ascher P. Glycine potentiates the NMDA response in cultured mouse brain neurons. Nature. 1987;325(6104):529-31.

32. Wei X, Walia V, Lin JC, Teer JK, Prickett TD, Gartner JJ, Davis S, Program NCS, Stemkehale K, Davies MA. Exome sequencing identifies GRIN2A as frequently mutated in melanoma. Nat Genet. 2011;43(5):442-6.

33. Balakrishnan A, Bleeker FE, Lamba S, Rodolfo M, Daniotti M, Scarpa A, Van Tilborg AAG, Leenstra S, Zanon C, Bardelli A. Novel somatic and Germline mutations in Cancer candidate genes in Glioblastoma, melanoma, and pancreatic carcinoma. Cancer Res. 2007:67(8):3545-50.

34. Burgess BL, Rao NP, Eskin A, Nelson SF, McCannel TA. Characterization of three cell lines derived from fine needle biopsy of choroidal melanoma with metastatic outcome. Mol Vis. 2011;17:607.

35. Cifola I, Pietrelli A, Consolandi C, Severgnini M, Mangano E, Russo V, De Bellis G, Battaglia C. Comprehensive genomic characterization of cutaneous malignant melanoma cell lines derived from metastatic lesions by wholeexome sequencing and SNP array profiling. PLoS One. 2013;8(5):e63597.

36. Elsey J, Bubley JA, Zhu L, Rao S, Sasaki M, Pollack BP, Yang L, Arbiser JL. Palladium based nanoparticles for the treatment of advanced melanoma. Sci Rep. 2019;9:3255.

37. Martincorena I, Raine KM, Gerstung M, Dawson K, Haase K, Van Loo P, Davies H, Stratton MR, Campbell PJ. Universal patterns of selection in cancer and somatic tissues. Cell. 2017;171(5):1029-41 e1021.

38. Weghorn D, Sunyaev S. Bayesian inference of negative and positive selection in human cancers. Nat Genet. 2017:49(12):1785.

39. Ke S, Shang S, Kalachikov S, Morozova I, Yu L, Russo JJ, Ju J, Chasin LA. Quantitative evaluation of all hexamers as exonic splicing elements. Genome Res. 2011;21(8):1360-74.

40. Xiong HY, Alipanahi B, Lee L, Bretschneider H, Merico D, Yuen RKC, Hua Y, Gueroussov S, Najafabadi HS, Hughes TR. The human splicing code reveals new insights into the genetic determinants of disease. Science. 2015; 347(6218):1254806
41. Lewis BP, Burge CB, Bartel DP. Conserved seed pairing, often flanked by adenosines, indicates that thousands of human genes are microRNA targets. Cell. 2005;120(1):15-20.

42. Mclaren WM, Gil L, Hunt S, Riat HS, Ritchie GRS, Thormann A, Flicek P, Cunningham F. The Ensembl variant effect predictor. Genome Biol. 2016; 17(1):122.

43. Petrovski S, Wang Q, Heinzen EL, Allen AS, Goldstein DB. Genic Intolerance to Functional Variation and the Interpretation of Personal Genomes. PLoS Genet. 2013;9(8).

44. Xin J, Mark A, Afrasiabi C, Tsueng G, Juchler M, Gopal N, Stupp GS, Putman TE, Ainscough BJ, Griffith OL. High-performance web services for querying gene and variant annotation. Genome Biol. 2016;17(1):91.

\section{Publisher's Note}

Springer Nature remains neutral with regard to jurisdictional claims in published maps and institutional affiliations.
Ready to submit your research? Choose BMC and benefit from:

- fast, convenient online submission

- thorough peer review by experienced researchers in your field

- rapid publication on acceptance

- support for research data, including large and complex data types

- gold Open Access which fosters wider collaboration and increased citations

- maximum visibility for your research: over $100 \mathrm{M}$ website views per year

At BMC, research is always in progress.

Learn more biomedcentral.com/submissions 